\title{
Crecimiento y Supervivencia de un Ensayo de Procedencias y Progenies de Roble (Nothofagus obliqua (Mirb.) Oerst.) de 21 Años de Edad en Pumillahue, Región de los Ríos, Chile.
}

\author{
Gutiérrez, Braulio ${ }^{1 \star} \&$ Navarrete, Mauricio ${ }^{2}$. \\ ${ }^{1}$ Ingeniero Forestal. Instituto Forestal, Sede Biobío. braulio.gutierrez@infor.cl \\ 2 Técnico Forestal. Instituto Forestal, Sede Biobío. \\ *Autor para correspondencia.
}

DOI: https://doi.org/10.52904/0718-4646.2021.547

Recibido: 05.08.2021; Aceptado 28.08.2021

\begin{abstract}
RESUMEN
Se evalúa el desempeño en crecimiento (altura, diámetro y volumen), supervivencia y rectitud de fuste de 29 procedencias de roble, anidadas en 10 zonas de procedencia representativas de la distribución natural de la especie en Chile. Los datos de la evaluación provienen de un ensayo de procedencias y progenies de 21 años de edad establecido en el predio Pumillahue (18H 691753,9 m E; 5611422,86 m S). Se caracteriza el desempeño general del ensayo y se evalúa el efecto de las procedencias y zonas de procedencia mediante análisis de varianza no paramétrico. Se detectan diferencias estadísticamente significativas entre procedencias para la supervivencia y variables de crecimiento, no así para la rectitud de fuste. El ensayo presenta un desempeño inferior al observado en un ensayo de comparación, no obstante, las procedencias Cuesta Lastarria y Cruces, de la zona 4C, presentan un comportamiento sobresaliente en crecimiento y supervivencia, lo que permite sugerirlas como idóneas y recomendables para su uso en plantaciones. Se constata que la zona de procedencia y la procedencia individual de mejor desempeño son las correspondientes a la zona de establecimiento del ensayo, confirmando los postulados respecto al uso preferente de semilla local en las iniciativas de plantación.
\end{abstract}

Palabras clave: Nothofagus obliqua, procedencia, crecimiento, supervivencia

\section{SUMMARY}

Growth performance (height, diameter and volume), survival and stem straightness of 29 oak provenances nested in 10 provenance areas representative of the natural distribution of the species in Chile are evaluated. The evaluation data come from a 21-year-old provenance and progeny trial established in the Pumillahue farm (18H $691753.9 \mathrm{~m} \mathrm{E;} 5611422.86 \mathrm{~m}$ $\mathrm{S})$. The general performance of the trial is characterized and the effect of provenances and provenance areas is evaluated by nonparametric analysis of variance. Statistically significant differences were detected between provenances for survival and growth variables, but not for stem straightness. The test presents a lower performance than that observed in a comparison test, however, the origins Cuesta Lastarria and Cruces, from zone 4C, present an outstanding performance in growth and survival, which allows to suggest them as suitable and recommended for use in plantations. It is found that the area of origin and the individual provenance with the best performance correspond to the area of establishment of the trial, confirming the postulates regarding the preferential use of local seed in planting initiatives.

Keywords: Nothofagus obliqua, provenance, growth, survival.

\section{INTRODUCCIÓN}

El roble (Nothofagus obliqua (Mirb.) Oerst.) es una especie forestal que ha concitado gran interés por su utilización, de hecho, sus bosques naturales fueron uno de los más cosechados en Chile, debido a su accesibilidad y fundamentalmente por al atractivo de su madera, que presenta valiosas propiedades mecánicas, durabilidad y admite diversos usos. Este mismo interés ha motivado su establecimiento en iniciativas de plantación, donde junto con coigüe ( $N$. dombeyi) y raulí ( $N$. alpina) han sido las especies más destacadas por sus tasas de crecimiento y el valor de sus maderas (Donoso y Soto, 2010). Los crecimientos registrados en plantaciones resultan superiores a los obtenidos en renovales de bosques naturales de la especie (Steuer, 2008), además las plantaciones presentan una buena poda natural, que permite plantar 
roble a densidades iniciales no muy altas y generar madera de mejor calidad (CONAF, 1998 cit. por Gutiérrez, 2004). Estos antecedentes coinciden con diversas declaraciones respecto a la potencialidad de roble y otros Nothofagus (raulí, coigüe), entre las especies nativas, para utilizarlos en el establecimiento de plantaciones comerciales. A pesar de lo anterior, esos resultados no han conseguido llamar del todo la atención de los propietarios o productores forestales, como para incluir mayores superficies plantadas con roble u otras destacadas especies nativas. Diversos motivos inciden en tal decisión, siendo una de los principales la mayor extensión de su rotación respecto a cultivos forestales exóticos, lo que desincentiva el establecimiento y manejo silvícola de plantaciones de especies nativas. Aun así, el desarrollo de estas plantaciones mejorará en la medida que se utilicen adecuadas técnicas silvícolas y material genético idóneo procedente de los orígenes adecuados para cada sitio de plantación, aspectos que deberán mejorar la supervivencia, crecimiento y productividad, y disminuir los periodos de rotación comercial.

Respecto de la idoneidad del origen del material genético usado para plantaciones, los ensayos de procedencia-progenie de especies forestales combinan la evaluación del origen del material (procedencia, región de procedencia) con el desempeño de progenies de árboles individuales procedentes de esos orígenes. Así, la evaluación de tales ensayos no solo permite determinar las mejores procedencias, sino que simultáneamente permite seleccionar los mejores individuos dentro de ellas (León, 2014; Landa et al., 2002). Por lo mismo, una importante aplicación práctica de estos ensayos es la de conocer el desempeño de los distintos materiales probados e identificar a los más apropiados para zonas equivalentes a aquella donde el ensayo está establecido; esto permite disponer de información objetiva para respaldar la elección del material genético que se usará en el establecimiento de una plantación.

Al respecto, en el presente documento se evalúa un ensayo de procedencias y progenies de roble de 21 años de edad, con el objetivo de conocer la variabilidad de desempeño, en términos de supervivencia, altura, diámetro, volumen y rectitud del fuste, de progenies de roble originarias de una treintena de procedencias de la especie, con la finalidad de orientar a propietarios e instituciones interesadas en plantaciones comerciales o de restauración, respecto a los orígenes y materiales genéticos apropiados para mejorar las perspectivas de rendimiento, perpetuación y regeneración de los árboles implantados.

\section{MATERIAL Y MÉTODO}

Se analiza la evaluación a los 21 años de edad de un ensayo de procedencias y progenies de roble ubicado en el predio Pumillahue, perteneciente a la Corporación Nacional Forestal, en la comuna de San José de la Mariquina, región de Los Ríos (18H 691753,9 m E; 5611422,86 m S), a una altitud de 241 msnm (Figura 1), en un área de clima templado lluvioso, con precipitación anual y temperaturas medias que se detallan en el Cuadro 1. El ensayo se compone de 5 bloques completos al azar donde se prueban progenies de roble, las que se anidan en 29 procedencias y 10 zonas de procedencia de acuerdo al detalle especificado en el cuadro 2. Las progenies involucradas en este ensayo se representan en cada bloque por una parcela de un árbol. La delimitación de las regiones de procedencia utilizada fue desarrollada por Vergara et al. (1998) y se resume en la Figura 2.

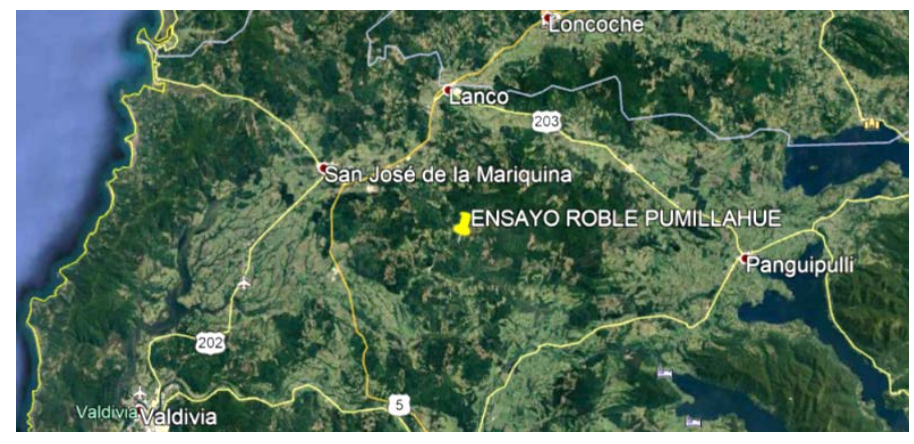

Figura 1. Ubicación Ensayo de Procedencias y Progenies de Roble.

Cuadro 1. Características Cimáticas del Área del Ensayo de Procedencias y Progenies de Roble. 


\begin{tabular}{lc}
\hline Variable climática & Valor \\
\hline Temperatura media estival & $16,1^{\circ} \mathrm{C}$ \\
\hline Temperatura mínima y máxima estival & $10,8^{\circ} \mathrm{C} ; 23,0^{\circ} \mathrm{C}$ \\
\hline Temperatura media invernal & $8^{\circ} \mathrm{C}$ \\
\hline Temperatura mínima y máxima invernal & $4,9^{\circ} \mathrm{C} ; 11,9^{\circ} \mathrm{C}$ \\
\hline PP normal anual & $1.978 \mathrm{~mm}$ \\
\hline (Fuente: MMA, 2016)
\end{tabular}

Cuadro 2. Regiones de Procedencia y Procedencias de las Progenies de Roble Evaluadas.

\begin{tabular}{|c|c|c|c|}
\hline $\begin{array}{l}\text { Región de } \\
\text { Procedencia }\end{array}$ & $\begin{array}{c}\text { Procedencia } \\
\text { (Punto de } \\
\text { Muestreo) }\end{array}$ & $\begin{array}{l}\text { Latitud } \\
{ }^{\circ} \mathrm{S}\end{array}$ & $\begin{array}{l}\text { Longitud } \\
{ }^{\circ} \mathbf{W}\end{array}$ \\
\hline \multirow{2}{*}{$1-C$} & Tiltil & $33^{\circ} 05^{\prime} 08^{\prime \prime}$ & $70^{\circ} 58^{\prime} 18^{\prime \prime}$ \\
\hline & Alhue & $33^{\circ} 57^{\prime} 56^{\prime \prime}$ & $71^{\circ} 01^{\prime} 41^{\prime \prime}$ \\
\hline \multirow{3}{*}{$2-C$} & Reserva Nac. Los Ruíles & $35^{\circ} 50^{\prime} 24^{\prime \prime}$ & $72^{\circ} 33^{\prime} 59^{\prime \prime}$ \\
\hline & Ninhue & $36^{\circ} 23^{\prime} 9^{\prime \prime}$ & $72^{\circ} 22^{\prime} 58^{\prime \prime}$ \\
\hline & Quirihue & $36^{\circ} 17^{\prime} 53^{\prime \prime}$ & $72^{\circ} 31^{\prime} 54^{\prime \prime}$ \\
\hline \multirow{4}{*}{$3-C$} & Pichipillahuén & $38^{\circ} 19^{\prime} 47^{\prime \prime}$ & $72^{\circ} 02^{\prime} 25^{\prime \prime}$ \\
\hline & Cerro Cayumanqui & $36^{\circ} 42^{\prime} 06^{\prime \prime}$ & $72^{\circ} 29^{\prime} 01^{\prime \prime}$ \\
\hline & Curanilahue & $37^{\circ} 28^{\prime} 28^{\prime \prime}$ & $73^{\circ} 20^{\prime} 52^{\prime \prime}$ \\
\hline & Lago Lanalhue & $37^{\circ} 50^{\prime} 50^{\prime \prime}$ & $73^{\circ} 20^{\prime} 52^{\prime \prime}$ \\
\hline \multirow{2}{*}{$4-C$} & Cuesta Lastarria $\left(^{*}\right)$ & $39^{\circ} 22^{\prime} 30^{\prime \prime}$ & $72^{\circ} 42^{\prime} 21^{\prime \prime}$ \\
\hline & Cruces & $39^{\circ} 31^{\prime} 32^{\prime \prime}$ & $73^{\circ} 04^{\prime} 13^{\prime \prime}$ \\
\hline \multirow{3}{*}{$5-C$} & Llancacura & $40^{\circ} 17^{\prime} 45^{\prime \prime}$ & $73^{\circ} 26^{\prime} 18^{\prime \prime}$ \\
\hline & Rio Negro & $40^{\circ} 47^{\prime} 8 \prime$ & $73^{\circ} 16^{\prime} 26^{\prime \prime}$ \\
\hline & Purranque & $40^{\circ} 52^{\prime} 02^{\prime \prime}$ & $73^{\circ} 14^{\prime} 09^{\prime \prime}$ \\
\hline \multirow{2}{*}{$6-D$} & Victoria & $38^{\circ} 12^{\prime} 14^{\prime \prime}$ & $72^{\circ} 10^{\prime} 23^{\prime \prime}$ \\
\hline & Quepe & $38^{\circ} 52^{\prime} 2^{\prime \prime}$ & $72^{\circ} 30^{\prime} 24^{\prime \prime}$ \\
\hline \multirow{3}{*}{ 7-D } & Malalhue & $39^{\circ} 30^{\prime} 55^{\prime \prime}$ & $72^{\circ} 32^{\prime} 06^{\prime \prime}$ \\
\hline & Futrono & $40^{\circ} 04^{\prime} 53^{\prime \prime}$ & $72^{\circ} 20^{\prime} 16^{\prime \prime}$ \\
\hline & Rupanco & $40^{\circ} 49^{\prime} 29^{\prime \prime}$ & $72^{\circ} 54^{\prime} 5^{\prime \prime}$ \\
\hline \multirow{4}{*}{$9-A$} & Reserva Nac. Ñuble & $36^{\circ} 55^{\prime} 30^{\prime \prime}$ & $71^{\circ} 12^{\prime} 37^{\prime \prime}$ \\
\hline & Ralco & $37^{\circ} 53^{\prime} 11^{\prime \prime}$ & $71^{\circ} 35^{\prime} 11^{\prime \prime}$ \\
\hline & Recinto & $36^{\circ} 51^{\prime} 11^{\prime \prime}$ & $71^{\circ} 39^{\prime} 43^{\prime \prime}$ \\
\hline & Santa Bárbara & $37^{\circ} 40^{\prime} 12^{\prime \prime}$ & $71^{\circ} 57^{\prime} 36^{\prime \prime}$ \\
\hline \multirow{4}{*}{$11-A$} & Galletué & $38^{\circ} 36^{\prime} 44^{\prime \prime}$ & $71^{\circ} 15^{\prime} 59^{\prime \prime}$ \\
\hline & Cunco & $38^{\circ} 52^{\prime} 02^{\prime \prime}$ & $71^{\circ} 51^{\prime} 11^{\prime \prime}$ \\
\hline & Lago Colico & $39^{\circ} 01^{\prime} 14^{\prime \prime}$ & $72^{\circ} 0^{\prime} 48^{\prime \prime}$ \\
\hline & Curarrehue & $39^{\circ} 22^{\prime} 53^{\prime \prime}$ & $71^{\circ} 32^{\prime} 00^{\prime}$ \\
\hline \multirow{2}{*}{$13-A$} & Choshuenco & $39^{\circ} 50^{\prime} 43^{\prime \prime}$ & $72^{\circ} 05^{\prime} 49^{\prime \prime}$ \\
\hline & Llifén & $40^{\circ} 11^{\prime} 08^{\prime \prime}$ & $72^{\circ} 15^{\prime} 00^{\prime}$ \\
\hline \multicolumn{4}{|c|}{ Testigo Mezcla de familias } \\
\hline \multicolumn{2}{|c|}{ Rellenos } & & \\
\hline
\end{tabular}




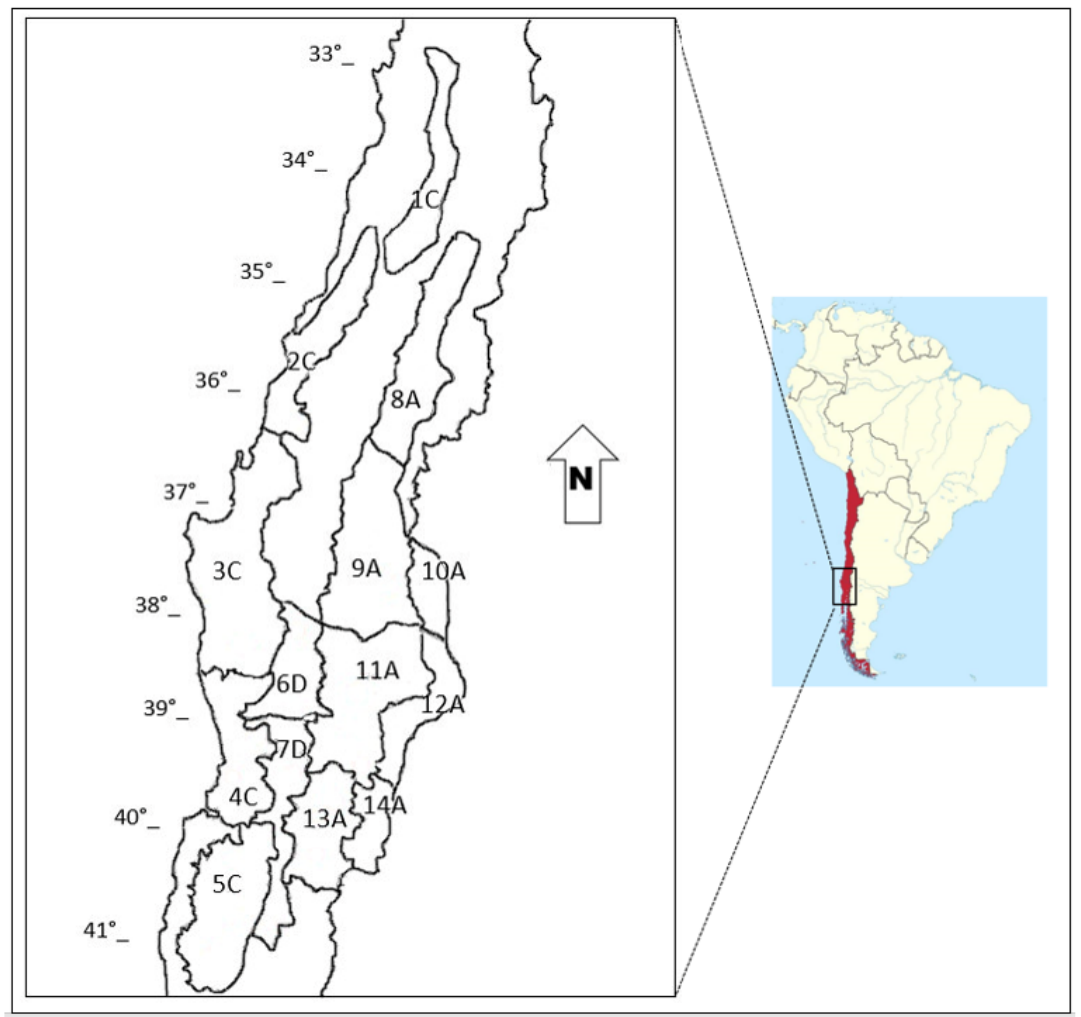

(Fuente: adaptado de Vergara et al., 1998)

Figura 2. Delimitación de las Regiones de Procedencia de las Progenies de Roble Evaluadas.

Las variables evaluadas para caracterizar el desempeño de las progenies de cada origen fueron: supervivencia (S), altura total $(\mathrm{H})$, diámetro a la altura del pecho (DAP), volumen (V) y rectitud de fuste (RF). La supervivencia (S) corresponde al porcentaje de árboles vivos respecto de los establecidos; la altura (ALT) es el valor total desde el suelo hasta el ápice del árbol; el DAP corresponde al diámetro del fuste a 1,3 metros del suelo; el volumen (V) corresponde al volumen sólido sin corteza, estimado con la función general desarrollada Puente et al. (1981) para renovales de roble (Expresión 1); la rectitud de fuste (RF) se evaluó con una escala cualitativa de cuatro niveles, donde 1 corresponde a los fustes menos rectos y 4 a los más rectos.

$$
V=0,05+0,00003151 * d^{2 *} h
$$

Donde:
V: volumen sólido sin corteza $\left[\mathrm{m}^{3}\right]$
d: diámetro [cm]
h: altura [m]

Se calculó los parámetros medios y de dispersión del ensayo para cada variable evaluada (S, H, DAP, V y $\mathrm{RF}$ ), se comprobó los supuestos de homogeneidad de varianzas y normalidad en la distribución de residuos, para posteriormente efectuar un análisis de varianza no paramétrico y de comparaciones múltiples usando la prueba de Kruskal y Wallis mediante el software estadístico Infostat 2015. Para efectos del análisis estadístico, el porcentaje de supervivencia se evaluó en unidades de Bliss, usando la transformación angular o del arcoseno, que resulta especialmente apropiada para análisis de varianza de variables en porcentajes o proporciones (Sokal y Rohlf, 1980; Mead et al., 2002). Posteriormente, para presentar los resultados la supervivencia se expresa en porcentaje como es lo habitual. 
Para efectos de comparar y caracterizar el desempeño de las distintas procedencias y zonas de procedencia se utilizó un índice simple que combina los parámetros de crecimiento y supervivencia en un solo valor (ecuación 2).

Este índice se usó para ordenar el desempeño de distintas entidades en orden decreciente y para efectuar comparaciones con los datos de un ensayo similar (ensayo de roble Huillilemu de 16 años de edad) cuya evaluación se obtuvo de Gutierrez (2020b).

$$
\mathrm{I}=\mathrm{IMA} \mathrm{A}_{\mathrm{V}} \text { S }
$$

Donde:

I= Índice de crecimiento y supervivencia para una procedencia o zona de procedencia

$\mathrm{IMA} v=$ Incremento medio anual en volumen de esa zona de procedencia o procedencia

$\mathrm{S}=$ Supervivencia de la zona de procedencia o procedencia

\section{RESULTADOS Y DISCUSIÓN}

\section{Resultados Generales}

A los 21 años de edad el ensayo de procedencias y progenies de roble presenta una supervivencia del $54,2 \%$ y valores medios de altura y dap de $16,1 \mathrm{~m}$ y $14,0 \mathrm{~cm}$, respectivamente (Cuadro 3).

Cuadro 3. Estadígrafos Descriptivos de las Variables Evaluadas en el Ensayo de Procedencia y Progenies Pumillahue a los 21 Años de Edad.

\begin{tabular}{|c|c|c|c|c|c|}
\hline Variable & $\begin{array}{c}\mathrm{S} \\
(\%) \\
n=737\end{array}$ & $\begin{array}{c}H \\
(m) \\
(n=729)\end{array}$ & $\begin{array}{c}\text { DAP } \\
(\mathrm{cm}) \\
(\mathrm{n}=729)\end{array}$ & $\begin{array}{c}V \\
\left(m^{3} / a r b\right) \\
(n=729)\end{array}$ & $\begin{array}{c}\text { RF } \\
(1-4) \\
(n=718)\end{array}$ \\
\hline Promedio & 54,2 & 16,1 & 14,0 & 0,190 & 2,2 \\
\hline D.E. & 25,3 & 3,7 & 6,1 & 0,130 & 0,9 \\
\hline Vmín & 0,0 & 5,2 & 2,0 & 0,051 & 1,0 \\
\hline Vmax & 100,0 & 25,4 & 32,8 & 0,824 & 4,0 \\
\hline
\end{tabular}

(D.E.= desviación estándar; Vmin= Valor mínimo; Vmax= Valor máximo)

En términos generales, la supervivencia $(S=54,2 \%)$ observada en Pumillahue resulta considerablemente inferior a la registrada a los 16 años en un ensayo similar, de procedencias y progenies de roble, establecido en el predio Huillilemu, en las cercanías de San José de la Mariquina, donde los árboles presentaban una supervivencia superior al 90\% (Gutiérrez, 2020b).

En cuanto a la rectitud de fuste, el valor medio de los árboles de Pumillahue (RF=2,2) es similar al obtenido en ensayo Huillilemu mencionado en párrafo anterior ( $R F=2,6)$ y considerablemente mejor que los observados en plantaciones de coigüe de similar edad, las que alcanzan valores de 1,4 y 1,7 en dos ensayos de procedencias y progenies de coigüe evaluados en Gutiérrez (2020a).

Respecto al crecimiento del ensayo Pumillahue, el valor medio de altura $(16,1 \mathrm{~m})$ registrados a los 21 años de edad, corresponde a un incremento medio anual de 0,76 m/año, el que resulta equivalente al incremento de 0,77 m/año registrado en ensayo Huillilemu a los 16 años (Gutiérrez, 2020b).

En el caso del DAP, el valor medio de Pumillahue (DAP= $14 \mathrm{~cm}$ ) equivale a un incremento medio anual de

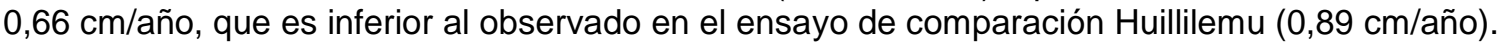

A pesar del menor desarrollo en DAP de los árboles de Pumillahue respecto a los de Huillilemu, sus incrementos medios de altura y DAP resultan similares a los informados en la bibliografía para otras plantaciones de roble (Cuadro 4). 
Cuadro 4. Incrementos Medios Anuales de Altura y Diámetro en Ensayo Pumillahue y otras Plantaciones de Referencia.

\begin{tabular}{ccll}
\hline $\begin{array}{c}\text { IMA Altura } \\
\text { (m/año) }\end{array}$ & $\begin{array}{c}\text { IMA DAP } \\
\text { (cm/año) }\end{array}$ & \multicolumn{1}{c}{ Plantación } & \multicolumn{1}{c}{ Fuente } \\
\hline 0,76 & 0,66 & Ensayo Pumillahue, 21 años & Este estudio \\
\hline 0,77 & 0,89 & Ensayo Huillilemu, 16 años & Gutiérrez,2020b \\
\hline $0,50-0,62$ & 0,40 & Llanquihue & Vita, 1977 \\
\hline 0,76 & 0,67 & Llanquihue & Meneses et al., 1991 \\
\hline 0,73 & 0,79 & Chiloé & Meneses et al., 1991 \\
\hline $0,51-0,50$ & $0,70-0,50$ & Frutillar, 31 y 39 años & Gutiérrez, 2004 \\
\hline & 0,57 & El Volcán, 34 años & Espinosa et al., 1988 \\
\hline & 0,70 & Frutillar, 14 años & Vita, 1977 \\
\hline 0,86 & Riñihue, 13 años & Donoso et al.,1993 \\
\hline 0,91 & 1,18 & Enco, 15 años & Donoso et al., 1993 \\
\hline 0,70 & & Palguín, 14 años & Donoso et al., 1993 \\
\hline 0,65 & Riñihue, 14 años & Donoso et al., 1995 \\
\hline 0,58 & Puñir, 39 años & Donoso et al., 1993 \\
\hline 0,88 & 7 tazas, 47 años & Donoso, 1988 \\
\hline & Panguipulli, 7 años & Donoso y Soto, 2010 \\
\hline
\end{tabular}

(Fuente: Elaboración propia con datos de Gutiérrez, 2004 y Steuer, 2008)

Los árboles del ensayo Pumillahue totalizan un volumen de $135,12 \mathrm{~m}^{3}$ en una superficie total de 1,088 ha, valores que equivalen a 670 árboles/ha, un volumen sólido sin corteza de 124,18 $\mathrm{m}^{3} / \mathrm{ha}$ y una productividad anual de $5,9 \mathrm{~m}^{3} / \mathrm{ha} / a n ̃ o$. Este valor resulta bajo respecto de los datos de referencia para la productividad anual de plantaciones de roble. En efecto, en la evaluación del ensayo de roble Huillilemu a los 16 años de edad se obtuvo un volumen de $154,5 \mathrm{~m}^{3} / \mathrm{ha}$ y una productividad anual media de $9,7 \mathrm{~m}^{3} / \mathrm{ha} / \mathrm{año}$ (Gutiérrez, 2020b). En ese mismo estudio se compilan referencias de distintos autores que indican una productividad anual esperada para plantaciones de roble del orden de $10 \mathrm{~m}^{3} / \mathrm{ha}$ año.

Las zonas de procedencia y las procedencias propiamente tales afectan en distinta forma a las variables evaluadas. La supervivencia experimenta diferencias estadísticamente significativas entre procedencias y entre zonas de procedencia; las variables de crecimiento ( $\mathrm{H}$, DAP y V) varían significativamente solo entre procedencias; en tanto que la rectitud de fuste no varía significativamente entre zonas de procedencia ni entre procedencias (Cuadro 5).

Cuadro 5: Significancia de las Diferencias entre Zonas de Procedencias y entre Procedencia para las Variables Evaluadas.

\begin{tabular}{lcccc}
\hline Variable & \multicolumn{2}{c}{$\begin{array}{c}\text { Supuestos ANDEVA } \\
\text { Paramétrico }\end{array}$} & $\begin{array}{c}\text { ANDEVA no Paramétrico } \\
\text { Kruskal y Wallis } \\
\text { p-values }\end{array}$ \\
\cline { 2 - 5 } & $\begin{array}{c}\text { Normalidad } \\
\text { Residuos }\end{array}$ & $\begin{array}{c}\text { Homogeneidad } \\
\text { Varianzas }\end{array}$ & $\begin{array}{c}\text { Zonas de } \\
\text { Procedencias }\end{array}$ & Procedencias \\
\hline Supervivencia (S) & si & no & $<0,001$ ** & $<0,001^{* *}$ \\
\hline Altura (H) & no & si & $0,0948 \mathrm{~ns}$ & $0,0360^{*}$ \\
\hline Diámetro (DAP) & no & si & $0,1362 \mathrm{~ns}$ & $0,0120^{*}$ \\
\hline Volumen (V) & no & no & $0,1236 \mathrm{~ns}$ & $0,0135^{*}$ \\
\hline Rectitud Fuste (RF) & no & si & $0,0931 \mathrm{~ns}$ & $0,3640 \mathrm{~ns}$ \\
\hline Diferencias estadísticas: * ${ }^{*}$ significativas; ${ }^{* *}$ muy significativas; ns no significativas &
\end{tabular}

\section{Resultados por Zonas de Procedencia}

En general el ensayo evidencia una diferenciación importante en la supervivencia de los árboles de las distintas zonas de procedencia. Por el contrario, no se observan diferencias significativas de crecimiento ni de rectitud de fuste. Así, considerando el efecto combinado de la supervivencia y el crecimiento se pueden ordenar las zonas de procedencia en función de su desempeño (Cuadro 6, Figura 3), lo que permite 
identificar como origen más adecuado a la zona de procedencia 4C, que es la zona donde se encuentra el ensayo de Pumillahue. Por el contrario, la zona con desempeño más deficiente corresponde a la 1C.

Al momento del muestreo para obtener las semillas que originaron las plantas evaluadas en el ensayo Pumillahue, la zona $1 \mathrm{C}$ representaba la distribución norte de roble, donde las poblaciones muestreadas corresponden a lo que se conocía como variedad macrocarpa o roble de Santiago y actualmente como Nothofagus macrocarpa, es decir a una especie diferente a Nothofagus obliqua.

Cuadro 6. Valores Medios de Supervivencia, Crecimiento y Rectitud de fuste, a los 21 Años de Edad, de las Zonas de Procedencia de Roble Evaluadas en Ensayo Pumillahue (ordenados decrecientemente en función del índice de crecimiento y supervivencia).

\begin{tabular}{|c|c|c|c|c|c|c|c|c|c|c|c|}
\hline $\begin{array}{c}\text { Zona de } \\
\text { Procedencia }\end{array}$ & \multicolumn{2}{|c|}{$\begin{array}{c}\mathrm{H} \\
(\mathrm{m})\end{array}$} & \multicolumn{2}{|c|}{$\begin{array}{l}\text { DAP } \\
(\mathrm{cm})\end{array}$} & \multicolumn{2}{|c|}{$\begin{array}{c}V \\
\left(m^{3}\right)\end{array}$} & \multicolumn{2}{|c|}{$\begin{array}{c}\text { RF } \\
(1-4)\end{array}$} & \multicolumn{2}{|c|}{$\begin{array}{c}\mathrm{S} \\
(\%)\end{array}$} & \multirow{2}{*}{\begin{tabular}{r}
\multicolumn{1}{c}{$\begin{array}{c}\text { Índice } \\
\text { (crec-sup) }\end{array}$} \\
0,838
\end{tabular}} \\
\hline $4 \mathrm{C}$ & 16,8 & a & 15,4 & $\mathrm{a}$ & 0,22 & $\mathrm{a}$ & 2,3 & $\mathrm{a}$ & 80,0 & $a$ & \\
\hline $7 \mathrm{D}$ & 16,5 & $a$ & 14,9 & $\mathrm{a}$ & 0,20 & $a$ & 2,1 & $a$ & 62,7 & $\mathrm{~b}$ & 0,597 \\
\hline $5 C$ & 16,2 & $a$ & 13,4 & $\mathrm{a}$ & 0,17 & $\mathrm{a}$ & 2,1 & $\mathrm{a}$ & 67,3 & $a b$ & 0,545 \\
\hline $13 \mathrm{~A}$ & 16,3 & $a$ & 14,3 & $\mathrm{a}$ & 0,19 & $\mathrm{a}$ & 2,1 & $\mathrm{a}$ & 57,0 & bc & 0,516 \\
\hline $3 C$ & 16,2 & $a$ & 14,1 & $\mathrm{a}$ & 0,19 & $\mathrm{a}$ & 2,0 & $\mathrm{a}$ & 53,1 & $\mathrm{bc}$ & 0,481 \\
\hline $6 \mathrm{D}$ & 15,6 & $a$ & 13,1 & $\mathrm{a}$ & 0,17 & $a$ & 2,4 & $a$ & 56,4 & bc & 0,457 \\
\hline $11 \mathrm{~A}$ & 15,9 & $a$ & 13,7 & $\mathrm{a}$ & 0,17 & $\mathrm{a}$ & 2,0 & $\mathrm{a}$ & 55,9 & $\mathrm{bc}$ & 0,452 \\
\hline $9 A$ & 15,7 & $a$ & 13,7 & $\mathrm{a}$ & 0,18 & $a$ & 2,2 & $a$ & 44,9 & $\mathrm{~cd}$ & 0,385 \\
\hline $2 \mathrm{C}$ & 16,4 & $a$ & 14,8 & $\mathrm{a}$ & 0,20 & $a$ & 2,3 & $a$ & 39,5 & cd & 0,376 \\
\hline TESTIGO & 16,0 & $a$ & 11,8 & $a$ & 0,19 & $a$ & 2,5 & $a$ & 40,0 & $\mathrm{bc}$ & 0,362 \\
\hline $1 \mathrm{C}$ & 13,9 & $a$ & 11,1 & $\mathrm{a}$ & 0,14 & $\mathrm{a}$ & 2,0 & $\mathrm{a}$ & 22,5 & $d$ & 0,150 \\
\hline PROM & 16,1 & & 14,0 & & 0,19 & & 2,2 & & 54,2 & & 0,490 \\
\hline
\end{tabular}

Letras distintas en la misma columna indican diferencias estadísticamente significativas entre medias

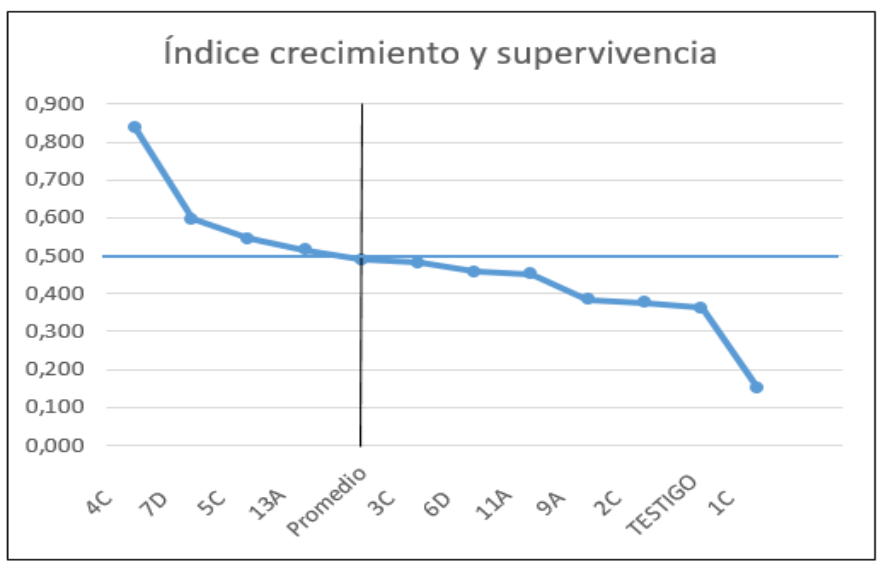

Figura 3. Ordenamiento de las Zonas de Procedencia Evaluadas en Ensayo de Roble Pumillahue en Función de su Desempeño Conjunto en Crecimiento y Supervivencia.

\section{Resultados por Procedencia}

A nivel de procedencias se detectan diferencias que poseen significación estadística para todas las variables, excepto para la rectitud de fuste (Cuadro 7).

La rectitud de fuste entre procedencias experimenta mayor variabilidad que la observada entre zonas de procedencia, aun así, esta diferenciación tampoco es estadísticamente significativa. La misma situación ha sido observada previamente en la evaluación de un ensayo de roble de 16 años (Gutiérrez 2020b) y otro de coigüe de 15 años de edad (Gutiérrez, 2017). 
Respeto a las variables de crecimiento ( $\mathrm{H}$, DAP y $\mathrm{V}$ ) en general se forman dos grupos altamente traslapados, donde solo las procedencias de desempeño extremo (mejores y peores) son diferentes entre sí, en tanto que las procedencias intermedias resultan relativamente homogéneas o similares entre ellas (Cuadro 7, Figura 4).

Cuadro 7. Valores Medios de Supervivencia, Crecimiento y Rectitud de Fuste, a los 21 Años de Edad, de las Procedencias de Roble Evaluadas en Ensayo Pumillahue.

\begin{tabular}{|c|c|c|c|c|c|c|c|c|c|c|}
\hline \multirow{2}{*}{$\begin{array}{c}\begin{array}{c}\text { Zona de } \\
\text { Procedencia }\end{array} \\
4 \mathrm{C}\end{array}$} & \multirow{2}{*}{$\begin{array}{l}\text { Procedencia } \\
\text { C LASTARRIA }\end{array}$} & $\begin{array}{c}\mathbf{H} \\
(\mathrm{m}) \\
\end{array}$ & $\begin{array}{l}\text { DAP } \\
(\mathrm{cm}) \\
\end{array}$ & \multicolumn{2}{|c|}{$\begin{array}{c}V \\
\left(m^{3}\right)\end{array}$} & \multicolumn{2}{|c|}{$\begin{array}{c}\text { RF } \\
(1-4)\end{array}$} & \multicolumn{2}{|c|}{$\begin{array}{c}\mathrm{S}^{*} \\
(\%) \\
\end{array}$} & \multirow{2}{*}{$\begin{array}{r}\begin{array}{c}\text { Índice } \\
\text { (crec-sup) }\end{array} \\
0,976\end{array}$} \\
\hline & & 17,5 a & $16,7 \quad a$ & 0,25 & $a$ & 2,2 & $a$ & 82,0 & $a$ & \\
\hline $4 \mathrm{C}$ & CRUCES & $16,1 \mathrm{ab}$ & $14,1 \mathrm{ab}$ & 0,19 & $\mathrm{~b}$ & 2,4 & $\mathrm{a}$ & 78,0 & $a$ & 0,706 \\
\hline $13 \mathrm{~A}$ & LLIFEN & 17,3 a & $15,9 \mathrm{ab}$ & 0,22 & $a$ & 2,2 & $a$ & 66,0 & $a b$ & 0,691 \\
\hline $3 C$ & LANALHUE & $16,6 \mathrm{ab}$ & $15,4 a b$ & 0,22 & $a$ & 1,9 & $a$ & 62,9 & $a b c$ & 0,659 \\
\hline $3 C$ & CURANILAHUE & $17,0 \mathrm{ab}$ & $15,4 a b$ & 0,22 & $\mathrm{a}$ & 2,0 & $a$ & 62,5 & $a b$ & 0,655 \\
\hline $7 \mathrm{D}$ & FUTRONO & $16,7 \mathrm{ab}$ & $15,9 a b$ & 0,22 & $\mathrm{a}$ & 2,0 & $\mathrm{a}$ & 60,0 & $a b c$ & 0,629 \\
\hline $7 \mathrm{D}$ & RUPANCO & $16,6 \mathrm{ab}$ & $14,7 \mathrm{ab}$ & 0,21 & $a b$ & 2,0 & $a$ & 62,0 & $a b c$ & 0,620 \\
\hline $11 \mathrm{~A}$ & COLICO & $16,6 \mathrm{ab}$ & $15,1 \mathrm{ab}$ & 0,20 & $a b$ & 2,1 & $\mathrm{a}$ & 63,3 & $a b c$ & 0,603 \\
\hline $5 \mathrm{C}$ & PURRANQUE & $16,7 \quad a b$ & $14,3 \mathrm{ab}$ & 0,18 & $\mathrm{~b}$ & 2,3 & $a$ & 70,0 & $a b$ & 0,600 \\
\hline $7 \mathrm{D}$ & MALALHUE & $16,3 a b$ & $14,3 \mathrm{ab}$ & 0,19 & $\mathrm{~b}$ & 2,2 & $\mathrm{a}$ & 66,0 & $a b$ & 0,597 \\
\hline $11 \mathrm{~A}$ & CURARREHUE & $17,1 \mathrm{ab}$ & $15,8 \mathrm{ab}$ & 0,21 & $a b$ & 2,2 & $a$ & 57,8 & $a b c$ & 0,578 \\
\hline $5 \mathrm{C}$ & RIO NEGRO & $16,3 a b$ & $14,0 \quad a b$ & 0,19 & $\mathrm{~b}$ & 2,2 & $a$ & 62,0 & $a b c$ & 0,561 \\
\hline $6 \mathrm{D}$ & QUEPE & $16,6 \mathrm{ab}$ & $14,7 \mathrm{ab}$ & 0,20 & $a b$ & 2,3 & $\mathrm{a}$ & 52,9 & $a b c$ & 0,504 \\
\hline $9 \mathrm{~A}$ & SANTA BARBARA & $16,0 \mathrm{ab}$ & $13,3 \mathrm{ab}$ & 0,17 & $\mathrm{~b}$ & 2,0 & $\mathrm{a}$ & 60,0 & $a b c$ & 0,486 \\
\hline $5 \mathrm{C}$ & LLANCACURA & $15,6 \mathrm{ab}$ & $12,0 \quad b$ & 0,14 & $\mathrm{~b}$ & 1,9 & $a$ & 70,0 & $\mathrm{a}$ & 0,467 \\
\hline $9 \mathrm{~A}$ & RN ÑUBLE & $15,8 a b$ & $15,5 \mathrm{ab}$ & 0,22 & $\mathrm{a}$ & 2,4 & $\mathrm{a}$ & 40,0 & $\mathrm{bc}$ & 0,419 \\
\hline $2 \mathrm{C}$ & QUIRIHUE & $16,6 \mathrm{ab}$ & $14,6 \mathrm{ab}$ & 0,20 & $a b$ & 2,2 & $\mathrm{a}$ & 43,2 & $a b c$ & 0,411 \\
\hline $6 \mathrm{D}$ & VICTORIA & $14,7 \quad a b$ & $11,6 \quad b$ & 0,14 & $\mathrm{~b}$ & 2,5 & $\mathrm{a}$ & 60,0 & $a b c$ & 0,400 \\
\hline $2 \mathrm{C}$ & RN LOS RUILES & $16,3 \mathrm{ab}$ & $15,2 \mathrm{ab}$ & 0,19 & $\mathrm{~b}$ & 2,5 & $\mathrm{a}$ & 44,0 & $a b c$ & 0,398 \\
\hline Testigo & MEZCLA FAM & $16,0 \mathrm{ab}$ & $11,8 \quad b$ & 0,19 & $\mathrm{~b}$ & 2,5 & $\mathrm{a}$ & 40,0 & $\mathrm{bc}$ & 0,362 \\
\hline $9 \mathrm{~A}$ & RECINTO & $15,3 a b$ & $13,8 \mathrm{ab}$ & 0,18 & $b$ & 2,2 & $\mathrm{a}$ & 42,2 & bc & 0,362 \\
\hline $3 C$ & PICHIPILLAHUEN & $15,9 a b$ & $12,8 \quad b$ & 0,15 & $b$ & 2,1 & $a$ & 50,0 & $a b c$ & 0,357 \\
\hline $11 \mathrm{~A}$ & CUNCO & $14,3 \quad b$ & $10,5 \mathrm{~b}$ & 0,13 & $\mathrm{~b}$ & 1,9 & $\mathrm{a}$ & 56,0 & $a b c$ & 0,347 \\
\hline $11 \mathrm{~A}$ & GALLETUE & $15,0 \mathrm{ab}$ & $12,8 \quad b$ & 0,16 & $\mathrm{~b}$ & 1,6 & $a$ & 42,9 & $a b c$ & 0,327 \\
\hline $2 \mathrm{C}$ & NINHUE & $16,2 \mathrm{ab}$ & $14,8 \mathrm{ab}$ & 0,20 & $a b$ & 2,3 & $a$ & 34,0 & C & 0,324 \\
\hline $13 \mathrm{~A}$ & CHOSHUENCO & $15,1 \mathrm{ab}$ & $12,2 \mathrm{~b}$ & 0,14 & $\mathrm{~b}$ & 2,0 & $\mathrm{a}$ & 48,0 & $a b c$ & 0,320 \\
\hline $9 \mathrm{~A}$ & RALCO & $15,7 \mathrm{ab}$ & $12,8 \quad b$ & 0,17 & $\mathrm{~b}$ & 2,3 & $a$ & 36,0 & C & 0,291 \\
\hline $3 C$ & CAYUMANQUI & $14,6 \mathrm{ab}$ & $11,7 \quad b$ & 0,13 & $b$ & 2,0 & $a$ & 37,1 & $\mathrm{bc}$ & 0,230 \\
\hline $1 \mathrm{C}$ & TILTIL & $14,5 \mathrm{ab}$ & $11,8 \quad b$ & 0,16 & $b$ & 1,8 & $a$ & 20,0 & $\mathrm{C}$ & 0,152 \\
\hline $1 \mathrm{C}$ & ALHUE & $12,7 \quad b$ & $9,7 \quad b$ & 0,09 & $b$ & 2,3 & $\mathrm{a}$ & 30,0 & $\mathrm{C}$ & 0,129 \\
\hline & PROM & 16,1 & 14,0 & 0,19 & & 2,2 & & 54,2 & & 0,490 \\
\hline
\end{tabular}

*Letras distintas en la misma columna indican diferencias estadísticamente significativas entre medias 


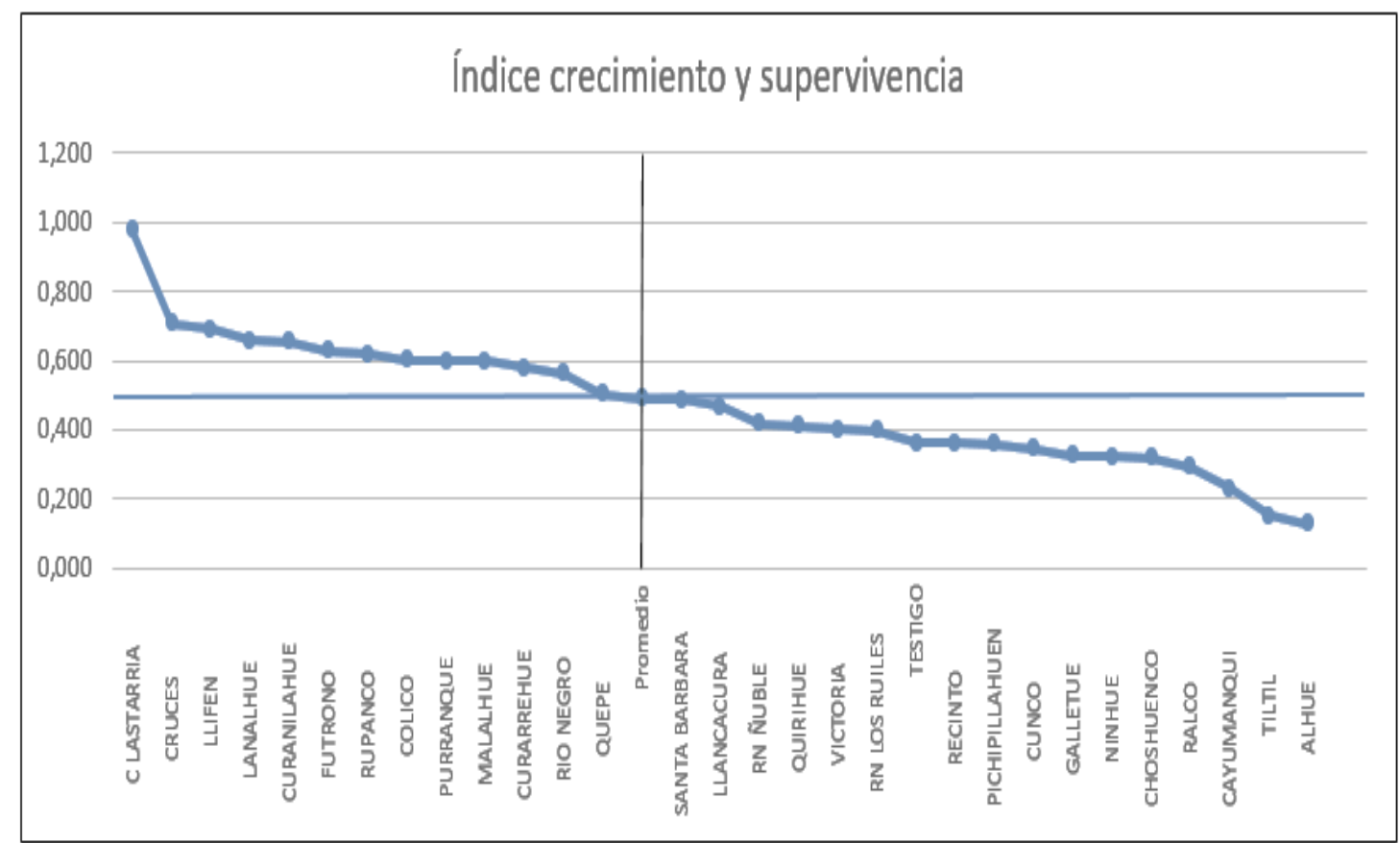

Figura 4. Ordenamiento de las Procedencias Evaluadas en Ensayo de Roble Pumillahue en Función de su Desempeño Conjunto en Crecimiento y Supervivencia.

En cuanto a la supervivencia, siguiendo la tendencia que ya se manifestaba a nivel de zonas de procedencia, en el caso de las procedencias también evidencia una diferenciación mayor que la exhibida por las variables de crecimiento.

El ordenamiento de las procedencias en función del índice combinado de supervivencia y crecimiento (Figura 4), permite identificar a las procedencias de la zona 4C, Cuesta Lastarria y Cruces, como las de mejor desempeño, destacándose el hecho que la procedencia Lastarria, que exhibe un comportamiento claramente superior a todas las restantes, es la procedencia local en el caso de Pumillahue.

La consistencia observada de la zona de procedencia y procedencia locales como las de mejor desempeño es concordante con la recomendación general de privilegiar el uso de material local en las iniciativas de plantación.

No obstante, en otras evaluaciones efectuadas en ensayos de roble (Gutiérrez, 2020b) no se ha verificado la misma coincidencia y los materiales locales no se han destacado en forma especialmente favorables entre los orígenes evaluados.

Consistentemente, las procedencias de la zona 1C (Tiltíl y Alhué) resultan las de peor desempeño en términos de supervivencia y crecimiento.

Respecto a las procedencias restantes, el gráfico de la Figura 4 ilustra claramente el comportamiento diferencial entre ellas y permite distinguir a las de mejor y peor desempeño, segregándolas en aquellas mejores y peores que el valor medio del ensayo.

Esta última situación se presenta también en el plano de la Figura 5, donde a nivel de zonas de procedencia se hace evidente que la zona local (4C) y las próximas a esta tienen mejor comportamiento en el sitio de Pumillahue que aquellas más lejanas.

En la Figura 5 se observa también que la diferencia entre procedencias tiene un efecto de carácter eminentemente latitudinal (N-S), mientras que longitudinalmente (O-E), no se manifiesta mayor influencia de las distintas posiciones fisiográficas (costa, depresión intermedia y andes). 


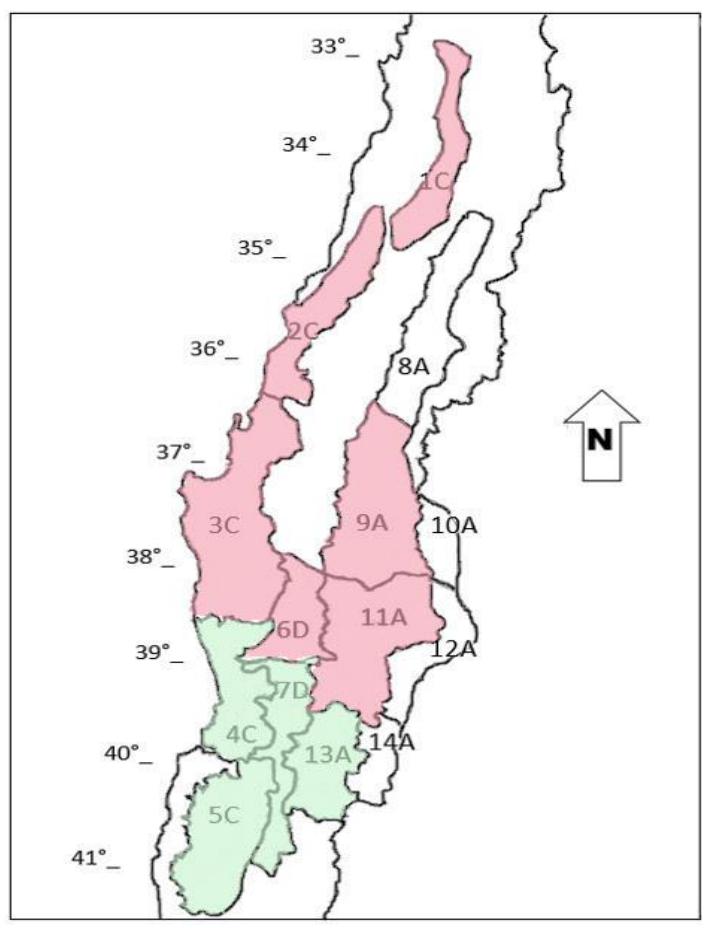

Figura 5. Orígenes de Roble con Desempeño Conjunto en Supervivencia y Crecimiento Superior (verde) e Inferior (rojo) a la Media del Ensayo Pumillahue. (Las zonas sin sombrear no fueron evaluadas en el ensayo)

\section{Comportamiento de Orígenes entre Sitios}

Una parte de las procedencias y zonas de procedencia establecidas en el ensayo Pumillahue se encuentran también representas en el ensayo homólogo Huillilemu, evaluado en Gutiérrez (2020b). Para estos orígenes comunes se efectuó una comparación de desempeño en términos del índice conjunto de supervivencia y crecimiento en cada ensayo. Los resultados de esta comparación se resumen en las Figuras 6 y 7, para las zonas de procedencia y para las procedencias, respectivamente.

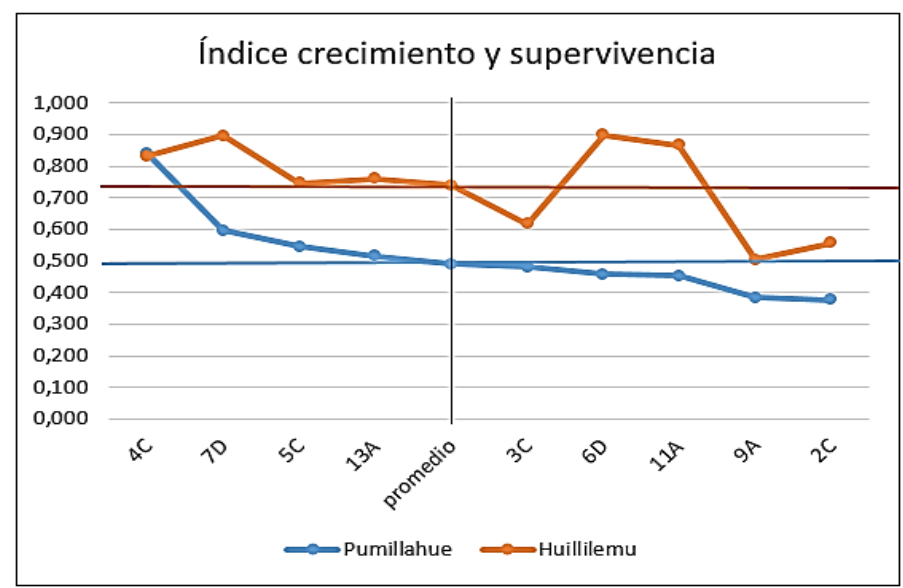

Figura 6. Comparación del Desempeño de Zonas de Procedencia de Roble Evaluadas en Ensayo Pumillahue y Huillilemu. 


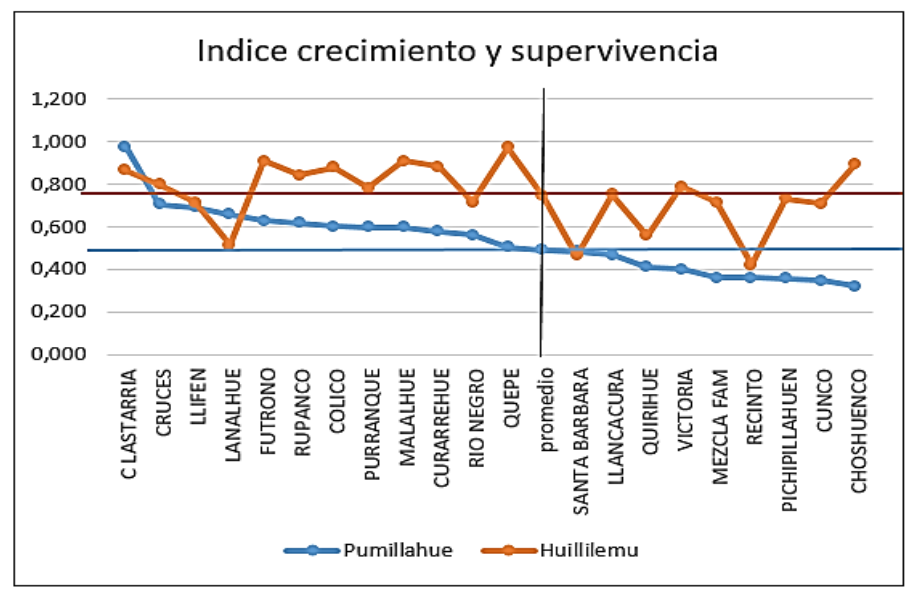

Figura 7. Comparación del Desempeño de Procedencias de Roble Evaluadas en Ensayo Pumillahue y Huillilemu.

El ensayo Pumillahue tiene un desempeño inferior al de Huillilemu, lo que se deriva principalmente de su menor supervivencia $(52,4$ vs $90,5 \%)$, y del menor valor de su incremento medio anual en diámetro $(0,66$ vs $0,89 \mathrm{~cm} / \mathrm{año})$, que reduce considerablemente su rendimiento en volumen respecto a Huillilemu $(5,9$ vs $\left.9,7 \mathrm{~m}^{3} / \mathrm{ha} / \mathrm{año}\right)$.

Esa situación se refleja en valores más bajos del índice conjunto de crecimiento y supervivencia para todas las zonas de procedencia comunes, así como para la mayoría de las procedencias individuales.

A nivel de zonas de procedencia, en ambos ensayos el peor desempeño lo presentan las zonas $2 \mathrm{C}$ y $9 \mathrm{~A}$, mientras que las zonas con mejores valores son distintas en cada ensayo (4C y 7D en Pumillahue; 6D y 7D en Huillilemu).

Aun así, las zonas que son superiores e inferiores a los promedios locales son, salvo dos excepciones, las mismas en los dos ensayos. A nivel de procedencias se presenta una variación similar. No existe coincidencia entre las peores y mejores procedencias en cada ensayo, pero en general se observa que aquellas que son mejores a la media de un ensayo, son las mismas que son mejores a la media en el ensayo de comparación; la misma situación ocurre para aquellas procedencias que son inferiores a los promedios locales.

\section{CONCLUSIONES}

A los 21 años de edad el ensayo de procedencias y progenies de roble de Pumillahue presenta una supervivencia relativamente baja y un rendimiento en volumen inferior al observado en ensayos homólogos y al registrado en la bibliografía.

No obstante, existen diferencias significativas entre procedencias para supervivencia, altura, diámetro y volumen, y el material genético de los mejores orígenes involucra una importante superioridad de volumen respecto a la media del ensayo.

Particularmente, los árboles de la procedencia Cuesta Lastarria y Cruces, de la zona 4C, presentan un comportamiento sobresaliente en crecimiento y supervivencia, lo que permite sugerirlos como idóneos y recomendables para su uso en plantaciones.

Se verifica que la zona de procedencia y la procedencia individual de mejor desempeño son las correspondientes a la zona de establecimiento del ensayo, confirmando los postulados respecto al uso preferente de semilla local en las iniciativas de plantación. 


\section{AGRADECIMIENTOS}

Los autores agradecen el apoyo prestado por la Corporación Nacional Forestal de la Región de los Ríos, en especial a los Sres. Neftalí Soto y Luis Riquelme por su permanente preocupación y apoyo.

\section{REFERENCIAS}

Donoso, P. (1988). Caracterización y proposiciones silviculturales para renovales de Roble (Nothofagus obliqua) y Raulí (Nothofagus alpina) en el área de protección "Radal 7 Tazas", VII Región. Bosque, 9(2): 103-114. https://doi.org/10.4206/bosque.1988.v9n2-07.

Donoso, P., Maureira, C., Barría, P. \& Hernández, E., 1995. Desarrollo inicial de plantaciones de Nothofagus en la provincia de Valdivia. Cuartas Jornadas Forestales Patagónicas. San Martín de los Andes. 18 p.

Donoso, P., Monfil, T., Otero, L. \& Barrales, L. (1993). Estudio de crecimiento de plantaciones y renovales manejados de especies nativas en el área andina de las provincias de Cautín y Valdivia. Ciencia \& Investigación Forestal, 7(2): 255-287. https://doi.org/10.52904/0718-4646.1993.188

Donoso, P. \& Soto, D. (2010). Plantaciones con especies nativas en el centro-sur de Chile: Experiencias, desafíos y oportunidades. Bosque Nativo, 47. Pp: 10-17.

Espinosa, M., Garcia, J. \& Peña, E. (1988). Evaluación del crecimiento de una plantación de Raulí (Nothofagus alpina (Poepp. et Endl) Oerst), de 34 años de edad. Agrociencia, 4(1): 67-74.

Gutiérrez, B. (2020 a). Influencia del origen de las semillas en el desempeño de coigüe (Nothofagus dombeyi (Mirb.) Oerst.) en la costa y precordillera de la región de Los Ríos. Ciencia \& Investigación Forestal, 26(2): 31-43. https://doi.org/10.52904/0718-4646.2020.531.

Gutiérrez, B. (2020 b). Desempeño de progenies, procedencias y regiones de procedencia de roble (Nothofagus obliqua). Ciencia \& Investigación Forestal, 26(3): 33-49. https://doi.org/10.52904/0718-4646.2020.536.

Gutiérrez, B. (2017). Evaluación de crecimiento y forma de fuste de un ensayo de procedencias y progenies de coihue (Nothofagus dombeyi (Mirb.) Oerst.) de 15 años de edad. Ciencia \& Investigación Forestal 23(3): 31-42. https://doi.org/10.52904/0718-4646.2017.484.

Gutierrez, N. (2004). Evaluación de crecimiento y rendimiento volumétricos en ensayos de plantación de Nothofagus obliqua y Nothofagus alpina al aplicar intervenciones silvícolas. Memoria para optar al título de Ingeniero Forestal. Universidad de Chile, Facultad de Ciencias Forestales. Santiago. 69 p.

Landa, J., Mendizábal, L. del C., Ramírez, E. \& Méndez, M. (2002). Establecimiento de tres ensayos de procedencia/progenie de Pinus teocote Schl. et Cham. En el estado de Veracruz. Foresta Veracruzana, 4(2): $17-22$.

León, N. (2014). Análisis de ensayos de procedencia-progenie de Dipteryx panamensis (Pittier) Record \& Mell, en la Zona Norte y Sur de Costa Rica. Tesis de Licenciatura en Ingeniería Forestal. Instituto Tecnológico de Costa Rica. Escuela de Ingeniería Forestal. Cártago, Costa Rica. 78 p.

Mead, R., Curnow, R.N. \& Hasted, A.M. (2002). Statistical methods in agriculture and experimental biology. $3^{\mathrm{a}}$ ed., Ed. Chapman \& Hall/CRC. Boca Raton, FL. 472 p.

Meneses, M., Paredes, G. \& Núñez, P. (1991). Opciones silviculturales para el manejo y utilización del bosque Siempreverde, Décima Región desde Río Bueno al sur. Informe de Convenio № 184. Informe Final. Universidad Austral de Chile, Facultad de Ciencias Forestales. Valdivia, Chile. 103 p.

MMA. (2016). Elaboración de una base digital del clima comunal de Chile: línea base (1980-2010) y proyección al año 2050. Información para de Desarrollo Productivo Ltda. Informe Final. 16 junio, 2016. Santiago. 142 p.

Puente, M., Peñaloza, R., Donoso, C., Paredes, R., Núñez, P., Morales, R. \& Engdahl, O. (1981). Estudio de raleos y otras técnicas para el manejo de renovales de raulí y roble. Instalación de ensayos de raleo. Proyecto CONAF/PNUD/FAO-CHI/76/003. Documento de trabajo No41. $47 \mathrm{p}$.

Sokal, R. \& Rohlf, F.J. (1980). Introducción a la bioestadística. Edición española. Editorial Reverte S.A. Barcelona, España.

Steuer, H. (2008). Evaluación de Raleos en un Renoval de Raulí (Nothofagus alpina Poepp.et Endl.) y Roble (Nothofagus obliqua (Mirb) Oerst) en la Provincia de Malleco, IX Región. Tesis Ingeniería Forestal. Universidad Austral de Chile, Facultad de Ciencias Forestales. Valdivia, Chile. 58 p. 
Vergara, R., Ipinza, R., Donoso, C. \& Grosse, H. (1998). Definición de zonas de procedencias de roble (Nothofagus obliqua (Mirb.) Oerst.) y raulí (Nothofagus alpina (Poep. et Endl) Oerst). Estado de avance. En: Primer Congreso Latinoamericano IUFRO, El manejo Sustentable de los Recursos Forestales, Desafío del Siglo XXI. Valdivia. 22-28 noviembre, 1998.

Vita, A. (1977). Crecimiento de algunas especies forestales en el Arboreto del Centro Experimental Frutillar. X Región. Boletín Técnico $N^{\circ}$ 47. Universidad de Chile, Facultad de Ciencias Forestales. Santiago. 16 p. 
Ciencia \& Investigación Forestal Vol. 27 № 2. Agosto 2021 